\title{
Postoperative Thoracic Epidural Analgesia: Adverse Events from a Single-Center Series of 3126 Patients
}

\section{Alberto Manassero (D) \\ Matteo Bossolasco (D) \\ Mattia Carrega \\ Giuseppe Coletta (ID}

Department of Emergency and Critical Care, Division of Operating Room Management, S. Croce e Carle Hospital, Cuneo, Italy
Correspondence: Alberto Manassero C.so 4 Novembre 12, CN 12100, Italy Tel +390171642025

Fax+390171642010

Email manassero.al@ospedale.cuneo.it
This article was published in the following Dove Press journal: Local and Regional Anesthesia

Purpose: Thoracic epidural analgesia (TEA) has been shown to reduce postsurgical morbidity and mortality; nevertheless, major and minor complications can occur. We report our 10-year experience with TEA and incidence of complications.

Patients and Methods: Patients received continuous infusion TEA $(0.2 \%$ ropivacaine and $2 \mu \mathrm{g} \mathrm{ml}^{-1}$ fentanyl) to control postoperative pain. Every 8 hours, the acute pain service recorded the analgesia regimen and occurrence of side effects. The initial infusion rate was tapered daily in response to improvement in pain symptoms or occurrence of side effects.

Results: A total of 3126 patients received TEA. The median age was 65 years (range, 18-94) and the duration of catheter placement was 3.5 days (range, 2-8). Three major complications were identified (1:1042): two subarachnoid blocks and one epidural abscess which led to permanent sequela (1:3126). Minor complications were hypotension $(4.8 \%)$, pruritus $(4.4 \%)$, accidental catheter removal (3.7\%), insertion site inflammation $(2.5 \%)$, motor weakness $(2.0 \%)$, postoperative nausea and vomiting $(1.8 \%)$, catheter disconnection $(1.9 \%)$, catheter occlusion $(0.3 \%)$, post-dural puncture headache $(0.5 \%)$, and catheter fragment retention $(0.06 \%)$, which were the reasons for a $7.4 \%$ rate of early discontinuation of epidural analgesia. No occurrence of epidural hematoma, local anesthetic systemic toxicity, and cardiovascular/respiratory depression was recorded.

Conclusion: Postoperative TEA is an advanced technique that poses certain difficulties that can subvert its great potential. While serious complications were rare, minor complications occurred more often and affected the postoperative course negatively. A risk/benefit evaluation of each patient should be done before employing the technique.

Keywords: thoracic epidural, postoperative pain, epidural analgesia, spinal epidural abscess, epidural catheter

\section{Introduction}

Thoracic epidural analgesia (TEA) has been shown to provide excellent analgesia and improve outcomes after many surgical procedures. ${ }^{1,2}$ A widely practiced analgesic technique, TEA is generally considered safe, though complications have been reported more in perioperative settings than in either chronic pain management or labor analgesia. ${ }^{3}$ Assessment and management of TEA-related adverse effects are essential for monitoring the perioperative course. Furthermore, reliable data on complications rate is key to guiding clinical decision making, adopting protective measures to prevent complications and their sequelae, and providing adequate information during the consent discussion with patients. Risk-benefit 
assessment has become foundational in routine practice owing to the increasing debate surrounding the role of TEA. ${ }^{4}$

The aim of this study was to investigate the incidence of major and minor TEA-related adverse effects over 10 years of experience.

\section{Patients and Methods}

The medical records of patients who received postoperative TEA in the surgery wards at S. Croce e Carle Cuneo City Hospital, between September 2009 and August 2019, were included in this prospective data collection. Patients received epidural analgesia after having given written informed consent for procedure and data management. Exclusion criteria were: patient refusal, coagulation disorders, generalized sepsis, known allergy to local anesthetics, and anatomical abnormalities of the spinal column. The data extracted from the medical records (age, sex, type of surgery, duration and quality of treatment, adverse events or unusual findings), were entered anonymized in the institutional encrypted database and then analyzed in an aggregate manner. This prospective data collection and analysis was conducted as a postoperative pain management "quality improvement" initiative, approved by the Ethics Committee of the S. Croce e Carle Hospital (protocol number 10/2020) and conducted in accordance with the Declaration of Helsinki.

Epidural catheters were placed in the operating theatre either immediately before induction of anesthesia or, to shorten anesthesia time, the afternoon of the day before surgery in patients scheduled for the first operating room time slot in the morning. Enoxaparin (40 mg) was given subcutaneously about $12 \mathrm{~h}$ before and after epidural catheter insertion. Antiplatelet therapy with aspirin was stopped 5 days before catheter insertion and therapy with clopidogrel and ticlopidine was stopped 7 or 10 days before. Platelet count $>80,000$ and coagulation times were ascertained to be within the normal range before catheter insertion and removal.

Each anesthesiologist followed an established institutional procedure for catheter insertion, using maximum barrier precaution and 3-step skin disinfection with an iodo-povidone solution. With the patient sitting upright, an epidural 20G 3-hole catheter with a soft atraumatic tip was inserted via an 8-cm 18G Tuohy needle (Perifix ${ }^{\circledR}$ Soft Tip 901 BBraun Melsungen, Germany) at a spinal level appropriate for the surgery (T5/6 for thoracic surgery and T8/9 or T9/10 for upper or lower abdominal surgery). The catheter was threaded into the epidural space for 5 to $7 \mathrm{~cm}$, secured with a transfixing silk suture to the underlying skin, and covered with a transparent, semipermeable sterile adhesive dressing (Tegaderm, 3M, St Paul, MN, USA). After a negative aspiration test for cerebrospinal fluid and blood, a dose of $2 \mathrm{~mL}$ lidocaine $2 \%$ was given to further exclude subarachnoid location of the catheter. The correct spread of the local anesthetic was assured by the loss of cold discrimination in the appropriate segments. Bacterial filters were used on all epidural catheters. All patients underwent general anesthesia and a local anesthetic bolus through the epidural catheter at the discretion of the anesthetist. An infusion of $0.2 \%$ ropivacaine with $2 \mu \mathrm{g}$ $\mathrm{ml}^{-1}$ fentanyl was started at 5 to $6 \mathrm{~mL} / \mathrm{h}$ before the end of surgery. The mixture was prepared aseptically in $300-\mathrm{mL}$ elastomeric pumps with an adjustable flow rate.

At the end of the surgery, patients were transferred to the postanesthesia care unit (PACU), where the efficacy of the analgesic regimen was checked and eventually adjusted based on a 4-point verbal rating scale (VRS, wherein a score of 0 indicates no pain at rest or on movement; 1 no pain at rest, slight pain on movement; 2 intermittent pain at rest, moderate on movement; and 3 continuous pain at rest, severe on movement). Patients were discharged to the wards if the VRS score was 0 to 1. The infusion regimen was continuous without the use of patient-controlled analgesia (PCA). The duration of epidural analgesia was planned as follows: $96 \mathrm{~h}$ (4 days) after pancreatectomy, esophagectomy, cystectomy, and thoracotomy surgery; b) 72 h (3 days) after nephrectomy, laparotomy for hysterectomy, liver, gastric, and colorectal surgery; c) 48 h (2 days) after laparoscopy surgery. The catheter was removed the day planned if patients reported satisfactory analgesia (VRS score 0 to 1 ) at a $3-\mathrm{mL} / \mathrm{h}$ infusion rate. Otherwise, epidural analgesia was continued after having evaluated it for clinical confirmation. The catheter was removed earlier if no longer required for analgesia. Pain episodes were treated with a rescue analgesic dose administered according to pain intensity as follows: VRS 1, paracetamol $1000 \mathrm{mg}$ i.v.; VRS 2, Ketorolac $30 \mathrm{mg}$ i.v.; VRS 3, morphine chlorhydrate $10 \mathrm{mg}$ i.m. If an unacceptable pain level (VRS 3) persisted, a top-up with lidocaine $1 \% 5 \mathrm{~mL}$ was carried out to ascertain the correct catheter position. If not, the epidural analgesia was discontinued and replaced with an i.v. elastomeric pump.

On the ward, a structured round was performed by the acute pain service (APS) every $8 \mathrm{~h}$ and whenever there were calls for pain management or infusion system problems. The 
analgesia regimen and side effects were recorded and scored: episodes of sedation ( 0 awake; 1 drowsy; 2 sleepy but easy to arouse, 3 sleepy and unrousable), nausea or vomiting (0 no nausea, 1 mild nausea, no antiemetic given, 2 moderate nausea, antiemetic given, 3 severe nausea and vomiting despite antiemetic), respiratory depression (ventilatory frequency $<8 \mathrm{bpm}$ ), hypotension (systolic arterial pressure $<80 \mathrm{~mm} \mathrm{Hg}$ after having excluded other common causes of hypotension after surgery) pruritus, leg numbness and weakness. Catheters were inspected daily and removed if signs of infection were observed (suppuration at the entry site). Catheters were also removed if disconnection from the filter was not immediately recognized. Motor impairment of any other drug-associated adverse event was managed by temporary reduction or discontinuation of infusion. Enoxaparin was withheld for at least $6 \mathrm{~h}$ after catheter removal. Within 24 $\mathrm{h}$ after catheter removal, the APS made at least two follow-up visits to check for any occurrences. The APS nurses compiled a tracking sheet for each patient. The tracking sheet contained information on epidural catheter insertion and daily evaluation of TEA efficacy and side effects. Major adverse events were defined as epidural hemorrhage or abscess, subarachnoid block, spinal cord-root injury leading to permanent motor deficits, TEA-associated cardiovascular/ respiratory collapse, and local anesthetic toxicity. Minor adverse events were defined as signs of local inflammation/ infection, episodes of hypotension, nausea or vomiting, sedation, pruritus, postdural puncture headache, motor weakness and numbness of the legs, catheter fragment retention, catheter occlusion, infusion system discontinuation, premature dislodgement. Neurological sequelae were considered permanent if they persisted for more than 6 months.

This study is descriptive with no prior hypothesis. Categorical variables are expressed as percentages and continuous variables as the median (min-max) because the distribution was non-normal. Statistical analysis was performed using GraphPad Prism for Windows, version 5.00 (GraphPad Software, San Diego, CA, USA).

\section{Results}

A total of 3126 patients ( $42 \%$ women and $58 \%$ men) received postoperative TEA (Figure 1). Table 1 presents the type of surgery and relative duration of TEA. The median age was 65 years (range, 18-94). The median duration of TEA was 3.5 days/ 84.0 hours (range, $2-8$ days). TEA was ended as planned with satisfactory pain control after tapering the infusion rate to $3 \mathrm{~mL} / \mathrm{h}$ in 1839 patients $(58.8 \%)$ and was extended in 1056 (33.8\%). TEA was discontinued because of complications in the remaining 231 (7.4\%) patients: accidental catheter removal in 116; catheter disconnected from the filter in 60; catheter occlusion in 10; local infection in 26; PDPH in 17; and subarachnoid block in 2 . Three major complications (1:1042) were recorded: two subarachnoid blocks and one epidural abscess, which led to permanent sequela (1:3126). Overall, 690 minor complications occurred in $431(13.8 \%)$ patients, in 111 (3.5\%) of which two or more complications occurred contemporaneously (Table 2 and Figure 2). No cases of epidural hematoma, local anesthetic systemic toxicity (LAST), TEA-associated cardiovascular/ respiratory collapse or sedation were noted.

\section{Details of Major Events Epidural Abscess}

One case of epidural abscess (EA) (1:3126-3,2:10.000) was recorded. $^{5}$ A 57-year-old man underwent a duodenopancreatectomy for an adenocarcinoma. The catheter entry point was examined daily, and no erythema or tenderness was noted. On postoperative day (POD) 2, the elastomeric pump was replaced. On POD 5, the patient developed pyrexia with no apparent cause. The epidural catheter was removed, and an erythematous swelling at the insertion site was noted. The same day, the patient started to complain of mild abdominal pain and distension. An abdominal computed tomography (CT) scan revealed two perihepatic fluid collections that were drained percutaneously. The patient became subpyretic. During the night of POD 12, the patient complained of mild back pain, which by the morning of POD 13 was associated with progressive bilateral leg weakness and difficulty walking. Sensory examination revealed decreased bilateral light touch up to the T4 dermatome. A magnetic resonance imaging (MRI) scan showed marked narrowing of the spinal canal and abnormal signals from the posterior epidural space between Th 6 and Th 8 (Figure 3). Within a few hours, complete paralysis of the lower extremities developed. An emergency decompressive laminectomy for drainage of the abscess was performed. Bacterial culture of purulent material produced methicillin-sensitive Staphylococcus aureus. Within 1 month, the patient recovered sensory deficit and motor power partially. At 1 year, the patient regained sphincter control but was unable to walk without the aid of crutches due to lower limb motor deficit.

\section{Subarachnoid Block}

Two cases of sudden onset of subarachnoid block were recorded $(1: 1563-6,4: 10.000)$. In both, the catheter was 


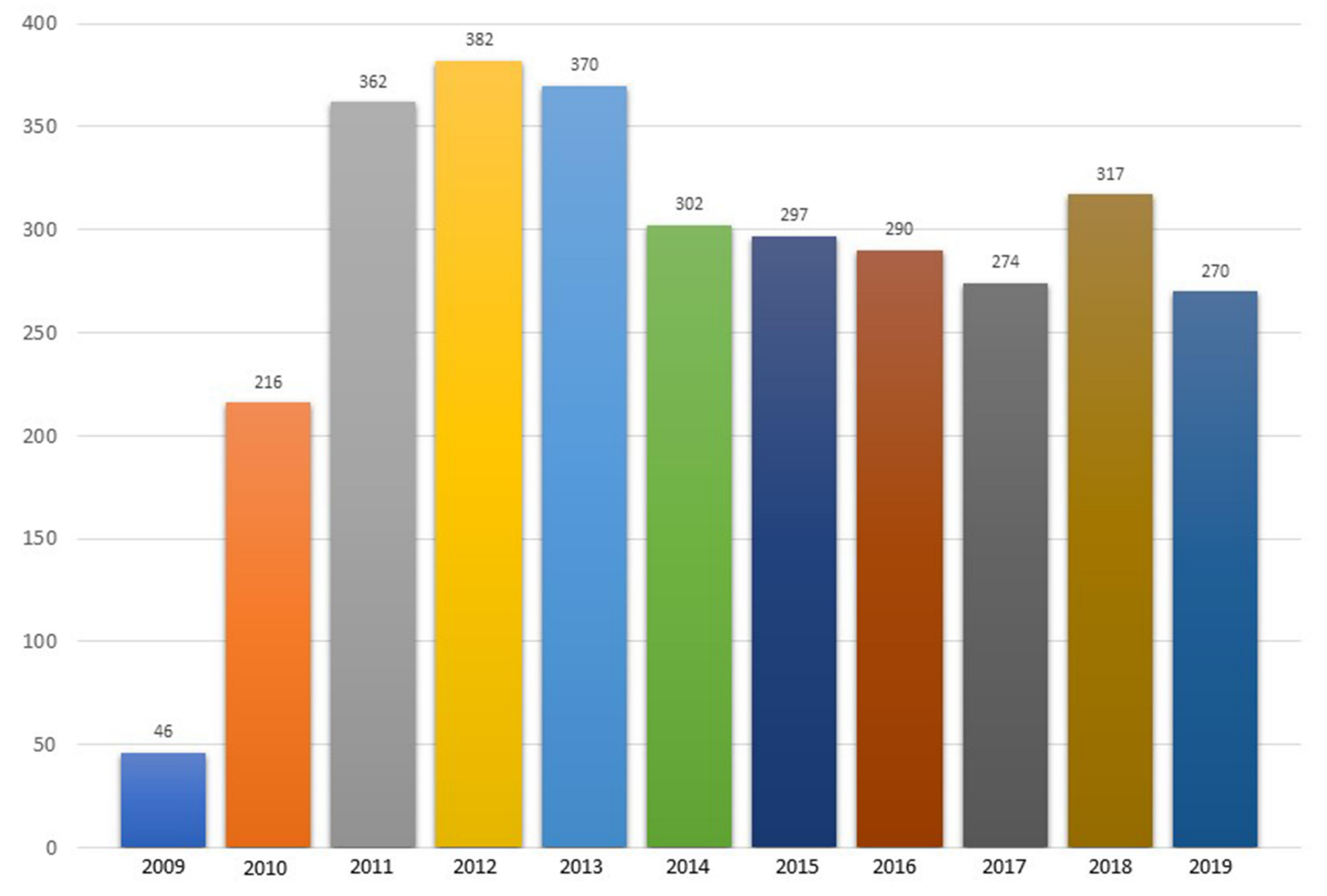

Figure I Cumulative yearly number of TEA performed.

implanted without difficulty, nothing could be aspirated from the needle or the catheter, and no detectable sensory or motor changes were noted after the $2-\mathrm{mL}$ lidocaine $2 \%$ test dose.

In the one case, a bolus of lidocaine $1 \% 5 \mathrm{~mL}$ administered during general anesthesia against surgical stress led to an unexpected drop in blood pressure and heart rate. As we suspected problems with the epidural catheter, no further anesthetic bolus was administered nor infusion started. Immediately after surgery, the patient was unable to move his legs, with sensory level of anesthesia at the T8 dermatome. Gentle aspiration through the catheter revealed cerebrospinal reflux. The block resolved within $1 \mathrm{~h}$ without sequelae. The catheter was removed; no headache developed. In the other case, no anesthetic bolus was administered during surgery and the infusion started at $5 \mathrm{~mL} / \mathrm{h}$ before the end of surgery. The patient was transferred to the ward after an uneventful surgery and postoperative period; the catheter was fully functional. Six hours later, the patient started to complain of progressive lower limb motor impairment and numbness up to the T10 dermatome. The infusion was stopped immediately, but no cerebrospinal fluid could be aspirated via the catheter. The block resolved within $2 \mathrm{~h}$ without sequelae. The catheter was removed; no headache developed.

\section{Discussion}

The side-effect profile of postoperative epidural analgesia varies across studies depending on catheter level (thoracic or lumbar), analgesia duration, drugs administered, method of delivery, and accuracy of the data collected. With this prospective study, we wanted to determine the incidence of complications attributed to thoracic epidural infusion delivered at a standard concentration of ropivacaine and fentanyl and under a standardized protocol of infusion and management at a single teaching hospital. In so doing we believe the reported data are consistent.

We identified as major complications two subarachnoid blocks and one EA. Despite the frequency of catheter colonization, neither a definitive causal relationship with epidural abscess has been established nor its incidence. Prospective audits have reported cases of epidural abscess 
Table I Types of Surgery and Duration of Thoracic Epidural Anesthesia

\begin{tabular}{|l|l|l|}
\hline Type of Surgery & No. (\%) & $\begin{array}{l}\text { Duration in Days } \\
\text { Median (Min-Max) }\end{array}$ \\
\hline Thoracotomy & $59(1.8)$ & $5.7(3-8)$ \\
Esophagectomy & $104(3.3)$ & $5.0(3-8)$ \\
Pancreatectomy & $132(4.3)$ & $4.2(3-6)$ \\
Cystectomy & $182(5.8)$ & $4.0(3-7)$ \\
Liver resection & $133(4.2)$ & $3.7(2-6)$ \\
Nephrectomy & $336(10.8)$ & $3.5(2-7)$ \\
Explorative laparotomy & $188(6.0)$ & $3.5(2-7)$ \\
Colon resection & $355(11.3)$ & $3.4(2-6)$ \\
Gastric resection & $177(5.6)$ & $3.4(2-6)$ \\
Hysterectomy & $41(1.3)$ & $3.4(2-7)$ \\
Rectal resection & $251(8.0)$ & $3.3(2-5)$ \\
Liver resection (LPS) & $22(0.8)$ & $2.9(2-4)$ \\
Gastric resection (LPS) & $50(1.5)$ & $2.9(2-6)$ \\
Rectal resection (LPS) & $129(4.2)$ & $2.8(2-7)$ \\
Colon resection (LPS) & $738(23.7)$ & $2.6(2-6)$ \\
Prostatectomy (open) & $197(6.3)$ & $2.5(2-5)$ \\
Nephrectomy (LPS) & $32(1.1)$ & $2.4(2-4)$ \\
All & $3126(100)$ & $3.5(2-8)$ \\
\hline
\end{tabular}

Abbreviation: LPS, laparoscopy.

with an incidence between 1 in 1368 to 1 in 7000 patients. $^{6-12}$ In the majority of the cases, there is no obvious cause and diagnosis is delayed. Presenting manifestations of spinal epidural abscess (SEA) may be insidious in nature: the classic clinical triad of back pain, fever, and neurological deficits are present in only a small percentage of patients. Early signs and symptoms of spinal epidural abscess can be non-specific and overlap with the common signs and symptoms of many other postoperative conditions. In our study, the delayed manifestation of back pain as a symptom, abdominal pain, and perihepatic fluid collections may have acted as confounding factors. ${ }^{13}$ The abdominal complications were often wrongly suspected as the cause of systemic infection. Regrettably, from the day of catheter removal (POD5) forward, the presence of pyrexia, laboratory findings, and, primarily, local signs of infection did not raise suspicion of an epidural infection and nor were swabs around the catheter insertion and catheter tip taken and cultured. A rigorous aseptic procedure was followed during catheter implant, but prolonged epidural catheterization, which lasted 5 days, including one elastomeric change, can have contributed to the development of infection. From this case forward, we changed several elements of our practice: the catheter remains in situ for a maximum of 4
Table 2 Thoracic Epidural Anesthesia-Related Complications

\begin{tabular}{|l|l|}
\hline No. (\%) & Major Complications \\
\hline $2(0.06)$ & Subarachnoid block \\
\hline (0.03) & Epidural abscess \\
\hline & Minor complications \\
\hline $150(4.8)$ & Hypotension \\
$137(4.4)$ & Pruritus \\
$116(3.7)$ & Accidental catheter removal \\
$80(2.6)$ & Inflammation at the entry site \\
$61(2.0)$ & Leg weakness \\
$60(1.9)$ & Catheter disconnection at the filter \\
$57(1.8)$ & Postoperative Nausea and Vomiting \\
$17(0.5)$ & Post Dural Puncture Headache \\
$10(0.3)$ & Catheter occlusion \\
$2(0.06)$ & Catheter fragment retention \\
\hline
\end{tabular}

days; if a prolonged epidural block is indicated, the risk/ benefit ratio is reassessed. Skin signs of infection and dressing damage are promptly reported to a supervisor of the postoperative pain management team. While erythema alone was not considered a sufficient reason for catheter removal, ${ }^{14}$ in the presence of suppurative signs, the decision to continue with epidural analgesia was made after considering the risk/benefit ratio case-by-case. Differently, catheter removal and bacteriological examination were performed whenever clinical symptoms indicated the possibility of infection of the epidural space.

Accidental subarachnoid block after epidural catheterization can be a life-threatening event, especially if it occurs in the ward where medical response time may be delayed. ${ }^{15-17}$ It is commonly attributed to catheter migration; however, as we used a soft-tipped epidural catheter in both cases, they could not by themselves have penetrated the dura. ${ }^{18}$ Probably, the catheters were accidentally placed in the subdural space in both cases, as neither the aspiration test nor the test dose was positive for subarachnoid placement. In the first case, we assume that the catheter moved into the subarachnoid space soon after its placement while in the second, the catheter was probably placed partly subdural and its distal segment remained across the dural membrane. Because it is a 3-holed catheter, some of the regularly injected solution (no bolus were given) flowed into the epidural space, providing regular epidural analgesia for about $6 \mathrm{~h}$, and some into the subdural space. The amount of solution that entered the subdural space gradually distended the arachnoid membrane enough to tear it and then suddenly create a subarachnoid 


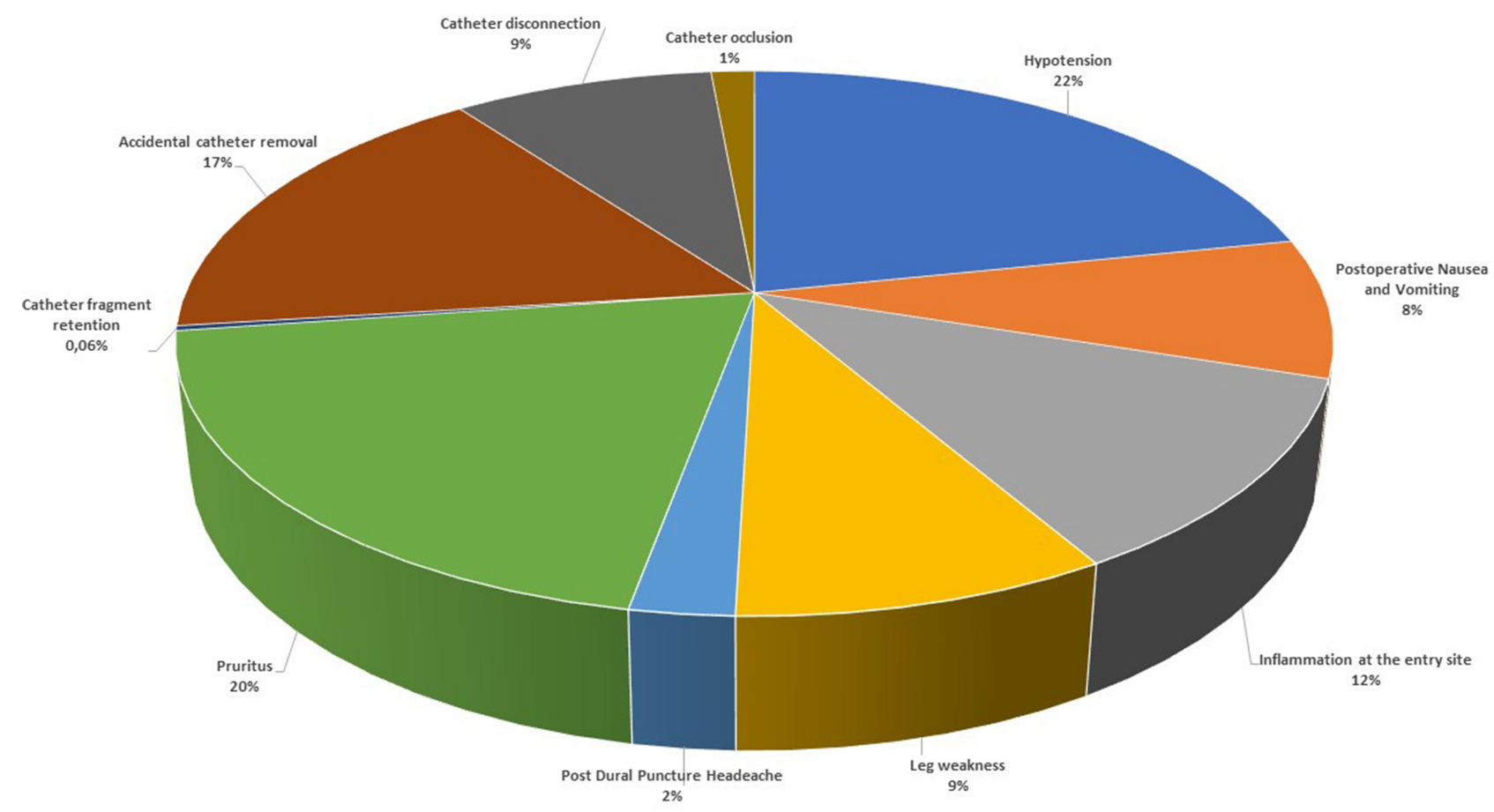

Figure 2 Distribution of complications expressed as percentage of the total.

block. Unfortunately, no MRI was performed in either case to determine the exact location of the catheter.

Hypotension was the most common minor complication (incidence, $4.8 \%$ ) and was within the range reported in previous studies (2.4-6.8\%). ${ }^{19-23}$ Hypotension is a clinically relevant postoperative side effect of TEA. Primarily due to blockade of the sympathetic nervous system and resulting in arterial and venous vasodilation with subsequent "functional" hypovolemia, it can delay early patient mobilization and, if corrected only with IV fluids, can cause fluid overload and reduce splanchnic perfusion, with potential negative effects on bowel anastomosis. ${ }^{24}$ Hypotension was recorded mainly in the immediate postoperative period, primarily when a laparoscopic technique was used (first night after surgery or the morning after). The symptom was corrected by reducing the epidural infusion rate; we linked this observation to an imbalance between nociception and antinociception.

The second most frequent complication was pruritus (incidence, 4.4\%); in 126 (92\%) patients its onset was within $24 \mathrm{~h}$ of the start of epidural infusion. The reported incidence of pruritus is dose-dependent (range, 1.8$16.7 \%$ ), making it the most common side effect of neuraxial opioids. ${ }^{19-23}$ In $33 \%$ of our patients who complained of pruritus, the elastomeric pump was replaced with an opioid-free one, which resolved the problem. In the remaining patients, it was sufficient to decrease the infusion rate.

We recorded 80 catheters with cutaneous signs of inflammation at the entry site (incidence, $2.6 \%$ ). The infection was suspected in 26 catheters because of either serous or purulent local discharge. The catheters were immediately removed, and a swab from the skin around the insertion site and from the tip of the catheter were cultured. The catheter tip and the skin around the insertion site were positive for same microorganism in 4/26 (15.3\%). All insertion site infections resolved shortly after catheter removal. Clinical signs of inflammation/ infection at the catheter entry site are variously defined and reported. Cameron reported a $2.8 \%$ incidence of insertion site infection among 7142 thoracic catheters with a mean insertion duration of 3.0 days compared to an incidence of $0.8 \%$ for 1068 lumbar catheters with a mean insertion duration of 2.1 days. There was a significant association between epidural insertion site infection and length of catheterization. ${ }^{6}$ Burstal reported an incidence of $5.3 \%$ for catheter site inflammation, with a highly significant difference in the incidence between a catheter left in situ for 3 days or less compared to those left for 4 or more days. ${ }^{22}$ Yuan found a strong relationship between bacterial colonization of the skin around the epidural catheter insertion site and tip segments of the catheter, 


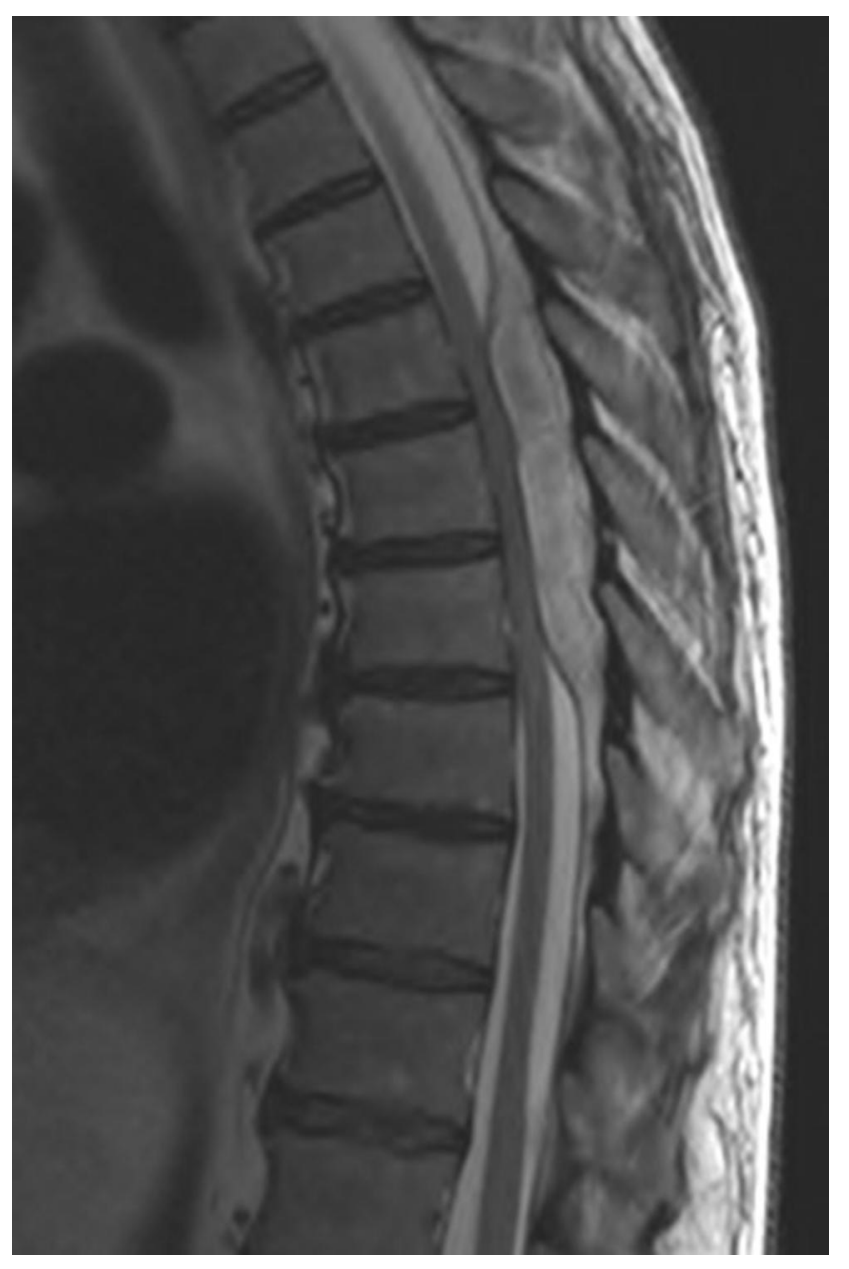

Figure $3 \mathrm{MRI}$ documenting the epidural abscess.

with a significant correlation between catheter tip colonization and dressing change and/or accidental catheter disconnection from the filter. ${ }^{25}$ Colonization of the catheter tip with the same organisms colonizing the skin, as in our 4 patients, reinforces the hypothesis that a common route for catheter colonization is via migration of bacteria along the catheter track or during its placement. This underscores the importance of employing aseptic practice during catheter placement and careful management to maintain the skin sterile around the catheter insertion site on the ward while limiting, whenever possible, dressing damage, accidental hub disconnection, bag changes, top-up infusate, and infusion for more than $96 \mathrm{~h}^{26}$

Motor weakness (Bromage score $>0$ ) and/or leg numbness was reported by $2.0 \%$ of patients. These symptoms correlate with the level of catheter placement, as the complication seems to be much lower when an epidural catheter is placed at the thoracic level. ${ }^{27}$ The incidence was similar to that reported by Ahmed (2.4\%) in a series of patients with the thoracic epidural catheter implanted at the T11-T12 interspace as a lower limit. ${ }^{28}$ The choice of ropivacaine $0.2 \%$, as well as the lower regimen of infusion $(\max 6 \mathrm{~mL} / \mathrm{h}$ ) and the absence of epidural bolus, may have resulted in the overall low incidence of motor impairment. As numbness was reported only as a discomforting symptom, lower limb motor weakness was a temporary restriction on patient mobilization. The highest frequency of motor impairment was seen in the first postoperative period and was successfully managed by decreasing the infusion flow rate without the need to stop the infusion.

Accidental dural puncture occurred in 58 patients $(1.8 \%)$ but postdural puncture headache (PDPH) developed in only $17(0.5 \%)$. The incidence of inadvertent dural puncture in our series is similar to the $1.5 \%$ and the $2.1 \%$ reported by Jackson $\mathrm{Su}^{29}$ and Deni, ${ }^{20}$ respectively, for their series of thoracic epidural catheters.

Postoperative nausea and vomiting (PONV) was recorded in $1.8 \%$ of patients; it can be a side effect of opioid administration itself. With fentanyl concentrations up to $10 \mu \mathrm{g} \mathrm{ml}^{-1}$, PONV rates range from 2.8 to $14.8 \% .{ }^{19,20,22,23}$ The incidence recorded in our series was lower than reported in the literature. A plausible explanation for this finding is the low fentanyl concentration.

Two cases of catheter fragment retention were recorded. In the first, the distal 5-mm soft material coating the catheter tip was retained; because of its small size, we were unable to confirm its position in the patient's body. In the second case, during catheter removal, the epidural catheter broke, and a fragment about $7 \mathrm{~cm}$ from the distal tip remained in the patient's body. The catheter fragment was visualized on a CT scan of the epidural space cephalad from the tip of the spinous process of the eleventh thoracic vertebra (Figure 4). Complications such as these have been described in previous case reports and studies. Recently, Hösslin reported a rate similar to ours. In accordance with current recommendations, we left both catheter fragments in situ. ${ }^{30-32}$

Accidental catheter removal occurred in $116(3.7 \%)$ patients, catheter disconnection at the filter in $60(1.9 \%)$, and catheter occlusion in $10(0.3 \%)$. Technical reasons for early discontinuation are a consistently reported problem in up to $19 \%$ of patients. ${ }^{19}$ Our data compare favorably with previously published series. ${ }^{21-23}$ We recorded a low rate of accidental catheter removal, similar to the 3.3\% reported by Hösslin, ${ }^{30}$ who adopted our technique of securing the catheter to the skin with a transfixing silk suture. In addition, to prevent outward movement, we also 


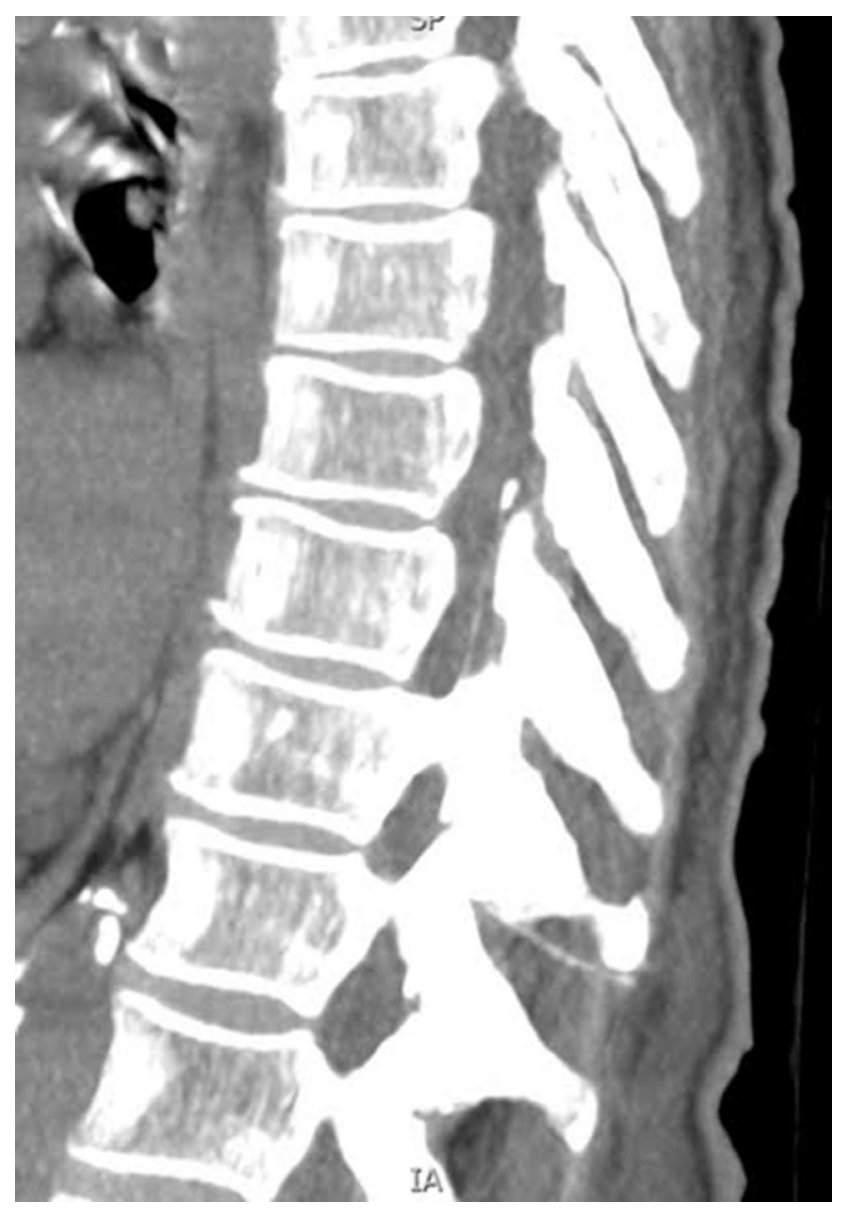

Figure 4 Thoracic spine CT showing the retained catheter fragment.

threaded the catheter into the epidural space for at least 5 to $7 \mathrm{~cm}^{27}$

The overall mean duration of TEA was 3.5 days (range, 2-8) for a total of 10,436 days, of which 1749 days (20\%) were more than those planned. In 1839 patients (58\%) TEA ended as planned with satisfactory pain control after tapering the infusion rate to $3 \mathrm{~mL} / \mathrm{h}$, while in 1056 (34\%) patients, it was continued past the scheduled time. The principal reason was that, at the planned day of catheter removal, either the analgesia level was not satisfactory or the coagulation profile was not within the normal range. In these cases, catheter removal was postponed for the next day. In some ways, prolonged duration of epidural analgesia may be viewed as another type of complication because it can delay recovery and increase the probability of catheter infection.

We recorded neither cases of epidural hematoma, nor of LAST; furthermore, the low concentration of fentanyl used may also explain the absence of dose-dependent opioid-related complications such as sedation and respiratory depression.

\section{Study Limitations}

The study population included patients undergoing continuous thoracic epidural infusion for postoperative analgesia; therefore, our findings can be translated only in part to patients undergoing patient-controlled or lumbar epidural analgesia. We cannot exclude the possibility of undetected adverse events.

\section{Conclusions}

Postoperative epidural analgesia is a sophisticated technique. It poses challenges that can subvert its great potential. Furthermore, adverse effects are more common than after other postoperative analgesia modalities. ${ }^{20}$ While serious complications happen rarely, less investigated minor complications may frequently occur and affect the postoperative course negatively, especially in the setting of an Enhanced Recovery After Surgery program. ${ }^{33,34}$ Some catheter-related complications are time dependent (eg, catheter infection and dislodgement), while others are drug dosage-dependent (eg, respiratory and conscious depression, hypotension, pruritus, PONV, motor weakness). Anesthetists need to be aware of these potentially avoidable complications. Taking into account that the epidural failed to provide satisfactory analgesia (at least one episode of VRS $\geq 2$ ) in $18 \%$ of patients, adding the incidence of complications (13.8\%) we can conclude that epidural analgesia was effective and safe in about two-thirds of patients. For this reason, the decision to use the technique must be carefully weighed when patients gain little analgesic benefit from an epidural (ie, laparoscopic surgery) or when less invasive regional analgesia is available. Since the completion of this survey and the related recognition of the potential problems associated with epidural analgesia, the number of patients receiving postoperative epidural analgesia at our institution decreased by $12 \%$ in 6 months.

\section{Disclosure}

The authors report no conflicts of interest in this work.

\section{References}

1. Pöpping DM, Elia N, Van Aken HK, et al. Impact of epidural analgesia on mortality and morbidity after surgery. Ann Surg. 2014;259 (6):1056-1067. doi:10.1097/SLA.0000000000000237

2. Block BM, Liu SS, Rowlingson AJ, Cowan AR, Cowan JA, Wu CL. Efficacy of postoperative epidural analgesia: a meta-analysis. JAMA. 2003;290(18):2455-2463. doi:10.1001/jama.290.18.2455

3. Cook TM, Counsell D, Wildsmith JAW. Major complications of central neuraxial block: report on the third national audit project of the Royal College of Anaesthetists. Br J Anaesth. 2009;102(2):179-190. doi:10.1093/bja/aen360 
4. Rawal N. Epidural technique for postoperative pain. Gold standard no more? Reg Anesth Pain Med. 2012;37(3):310-317. doi:10.1097/ AAP.0b013e31825735c6

5. Manassero A, Ugues S, Coletta G, Bugada D. Spinal epidural abscess: stay focused, stay tuned! A clinical report with negative neurological outcome from the "Italian Registry of Complications Associated with Regional Anesthesia-RICALOR.". Minerva Anestesiol. 2016;82(4):492-493.

6. Cameron CM, Scott DA, McDonald WM, Davies MJ. A review of neuraxial epidural morbidity: experience of more than 8000 cases at a single teaching hospital. Anesthesiology. 2007;106(5):997-1002. doi:10.1097/01.anes.0000265160.32309.10

7. Dahlgren N, Törnebrandt K. Neurological complications after anaesthesia. A follow-up of 18,000 spinal and epidural anaesthetics performed over three years. Acta Anaesthesiol Scand. 1995;39 (7):872-880. doi:10.1111/j.1399-6576.1995.tb04190.x

8. Pöpping DM, Zahn PK, Van Aken HK, Dasch B, Boche R, PogatzkiZahn EM. Effectiveness and safety of postoperative pain management: a survey of 18925 consecutive patients between 1998 and 2006 (2nd revision): A database analysis of prospectively raised data. Br J Anaesth. 2008;101(6):832-840. doi:10.1093/bja/aen300

9. Wang LP, Hauerberg J, Schmidt JF. Incidence of spinal epidural abscess after epidural analgesia. A national 1-year survey. Anesthesiology. 1999;91(6):1928-1936. doi:10.1097/00000542199912000-00046

10. Allegri M, Bugada D, Grossi P, et al. Italian registry of complications associated with regional anesthesia (RICALOR). An incidence analysis from a prospective clinical survey. Minerva Anestesiol. 2016;82 (4):392-402.

11. Davis DP, Wold RM, Patel RJ, et al. The clinical presentation and impact of diagnostic delays on emergency department patients with spinal epidural abscess. J Emerg Med. 2004;26(3):285-291. doi:10.1016/j.jemermed.2003.11.013

12. Pradilla G, Nagahama Y, Spivak AM, Bydon A, Rigamonti D. Spinal epidural abscess: current diagnosis and management. Curr Infect Dis Rep. 2010;12(6):484-491. doi:10.1007/s11908-010-0140-1

13. Bremer AA, Darouiche RO. Spinal epidural abscess presenting as intra-abdominal pathology: a case report and literature review. $J$ Emerg Med. 2004;26(1):51-56. doi:10.1016/j.jemermed. 2003.04.005

14. Darchy B, Forceville X, Bavoux E, Soriot F, Domart Y. Clinical and bacteriologic survey of epidural analgesia in patients in the intensive care unit. Anesthesiology. 1996;85(5):988-998. doi:10.1097/ 00000542-199611000-00005

15. Frohlich S. A rare case of epidural catheter migration? Minerva Anestesiol. 2012;78(7):858.

16. Reynolds F, Speedy HM. The subdural space: the third place to go astray. Anaesthesia. 1990;45(2):120-123. doi:10.1111/j.13652044.1990.tb14275.x

17. Elliott DW, Voyvodic F, Brownridge P. Sudden onset of subarachnoid block after subdural catheterization: a case of arachnoid rupture? $\mathrm{Br}$ J Anaesth. 1996;76(2):322-324. doi:10.1093/bja/76.2.322

18. Hardy PAJ. Can epidural catheters penetrate dura mater? An anatomical study. Anaesthesia. 1986;41(11):1146-1147. doi:10.1111/ j.1365-2044.1986.tb12968.x
19. Scott DA, Beilby D, McClymont C. Postoperative analgesia using epidural infusions of fentanyl with bupivacaine. A prospective analysis of 1014 patients. Anesthesiology. 1995;83(4):727-737. doi:10.1097/00000542-199510000-00012

20. Deni F, Greco M, Turi S, et al. Acute pain service: a 10-year experience. Pain Pract. 2019;19(6):586-593. doi:10.1111/papr.12777

21. Wigfull J, Welchew E. Survey of 1057 patients receiving postoperative patient-controlled epidural analgesia. Anaesthesia. 2001;56 (1):70-75. doi:10.1046/j.1365-2044.2001.01763-6.x

22. Burstal R, Wegener F, Hayes C, Lantry G. Epidural analgesia: prospective audit of 1062 patients. Anaesth Intensive Care. 1998;26 (2):165-172. doi:10.1177/0310057X9802600206

23. Liu SS, Allen HW, Olsson GL. Patient-controlled epidural analgesia with bupivacaine and fentanyl on hospital wards. Anesthesiology. 1998;88(3):688-695. doi:10.1097/00000542-199803000-00020

24. Gould TH, Grace K, Thorne G, Thomas M. Effect of thoracic epidural anaesthesia on colonic blood flow. Br J Anaesth. 2002;89 (3):446-451. doi:10.1093/bja/89.3.446

25. Yuan H-B, Zuo Z, Yu K-W, Lin W-M, Lee H-C, Chan K-H. Bacterial colonization of epidural catheters used for short-term postoperative analgesia microbiological examination and risk factor analysis. Anesthesiology. 2008;108(1):130-137. doi:10.1097/01. anes.0000296066.79547.f3

26. Brooks K, Pasero C, Hubbard L, Coghlan RH. The risk of infection associated with epidural analgesia. Infect Control Hosp Epidemiol. 1995;16(12):725-728. doi:10.2307/30141916

27. Königsrainer I, Bredanger S, Drewel-Frohnmeyer R, et al. Audit of motor weakness and premature catheter dislodgement after epidural analgesia in major abdominal surgery. Anaesthesia. 2009;64 (1):27-31. doi:10.1111/j.1365-2044.2008.05655.x

28. Ahmed A, Baig T. Incidence of lower limb motor weakness in patients receiving postoperative epidural analgesia and factors associated with it: an observational study. Saudi J Anaesth. 2016;10 (2):149-153. doi:10.4103/1658-354X.168806

29. Su J, Soliz JM, Popat KU, Gebhardt R. Complications of postoperative epidural analgesia for oncologic surgery: a review of 18,895 cases. Clin J Pain. 2019;35(7):589-593. doi:10.1097/AJP.0000000000000718

30. von Hösslin T, Imboden P, Lüthi A, Rozanski MJ, Schnider TW, Filipovic M. Adverse events of postoperative thoracic epidural analgesia. Eur J Anaesthesiol. 2016;33(10):708-714. doi:10.1097/ EJA.0000000000000446

31. Mitra R, Fleischmann K. Management of the sheared epidural catheter: is surgical extraction really necessary? J Clin Anesth. 2007;19 (4):310-314. doi:10.1016/j.jclinane.2006.11.005

32. Kasivisvanathan R, Sodhi M, Setty S. The broken epidural catheter: to remove or not to remove? Br J Hosp Med (Lond). 2012;73 (12):718. doi:10.12968/hmed.2012.73.12.718

33. Rosen DR, Wolfe RC, Damle A, et al. Thoracic epidural analgesia: does it enhance recovery? Dis Colon Rectum. 2018;61 (12):1403-1409. doi:10.1097/DCR.0000000000001226

34. Gustafsson UO, Scott MJ, Hubner M, et al. Guidelines for perioperative care in elective colorectal surgery: enhanced recovery after surgery $\left(\right.$ ERAS $\left.^{\circledR}\right)$ society recommendations: 2018. World J Surg. 2019;43(3):659-695.
Local and Regional Anesthesia

\section{Publish your work in this journal}

Local and Regional Anesthesia is an international, peer-reviewed, open access journal publishing on the development, pharmacology, delivery and targeting and clinical use of local and regional anesthetics and analgesics. The journal welcomes submitted papers covering original research, basic science, clinical studies, reviews \& evaluations, guidelines, expert opinion and commentary, case reports and extended reports. The manuscript management system is completely online and includes a very quick and fair peer-review system, which is all easy to use. Visit http://www.dovepress.com/testimonials. php to read real quotes from published authors. 